\title{
Proteomics study of serum exosomes from papillary thyroid cancer patients
}

\author{
Dan Luo',*, Shaohua Zhan ${ }^{1, *}$, Wenchao Xia', Liang Huang², Wei Ge1 and Tianxiao Wang ${ }^{3}$ \\ 'National Key Laboratory of Medical Molecular Biology \& Department of Immunology, Institute of Basic Medical Sciences, Chinese Academy of Medical \\ Sciences, Beijing, China \\ 2Department of Blood Transfusion, Tongji Hospital, Tongji Medical College, Huazhong University of Science and Technology, Wuhan, China \\ 3Department of Head and Neck Surgery, Key Laboratory of Carcinogenesis and Translational Research, Peking University Cancer Hospital \& Institute, \\ Beijing, China
}

Correspondence should be addressed to W Ge or T Wang: wei.ge@chem.ox.ac.uk or tianxiao_w2000@hotmail.com

*(D Luo and S Zhan contributed equally to this work)

\begin{abstract}
Lymph node metastasis (LNM) in papillary thyroid cancer (PTC) is related to increased risk of recurrence and poor prognosis. Tumour exosomes have been shown to be associated with metastasis of cancer cells. Therefore, we aim to identify the characteristics and biological functions of serum exosomes in lymph node metastases of PTC. We compared proteome profiles of serum-purified exosomes (SPEs) from PTC patients with LNM, PTC patients without LNM, and healthy donors, using a combination of liquid chromatography-tandem mass spectroscopy analyses and tandem mass tag label quantitation analysis. We identified 1569 proteins by two or more unique peptides. Compared with the SPEs of PTC patients without LNM, we found 697 differentially expressed proteins in the SPEs of PTC patients with LNM. Our results revealed overexpression of specific proteins with well-established links to cancer cell metastasis, such as SRC, TLN1, ITGB2 and CAPNS1. Consistent with mass spectrum results, we performed Western blot to detect the expression of these proteins in individual sample. Biological pathway analyses showed that integrin signalling was aberrantly activated in the SPEs of PTC patients with LNM compared to those without LNM. Our study reveals that SPEs of PTC patients with lymph node metastases promote BHT101 thyroid cancer cell invasiveness, but have no apparent influence on cell migration. In the serum exosomes of PTC patients with LNM, integrin-associated proteins are obviously upregulated. These proteomic findings will contribute to elucidation of the pathophysiological functions of tumour-derived exosomes.
\end{abstract}

\section{Key Words}

- papillary thyroid cancer (PTC)

- lymph node metastasis (LNM)

- serum-purified exosomes (SPES)

- proteomic analysis

\section{Introduction}

Worldwide, thyroid cancer is the most common endocrine malignancy, accounting for $2.1 \%$ of all cancer diagnoses (Kitahara \& Sosa 2016). In recent decades, the incidence of thyroid cancer has increased substantially throughout much of the world (Cabanillas et al. 2016). The most common type of thyroid cancer is papillary thyroid cancer (PTC), comprising almost $80 \%$ of all cases (Schneider \& Chen 2013). Differentiated PTC usually has an excellent prognosis with 5 -year survival $>97 \%$ (Howlader et al. 2012). However, retrospective reviews show that lymph 
node metastases are one of the most significant risk factors that affect recurrence and prognosis (Beasley et al. 2002, Schneider et al. 2013). The precise molecular mechanisms promoting progression of lymph node metastases of PTC have not been well elucidated.

Recently, tumour-derived exosomes have been demonstrated to be involved in cancer metastases (Liu et al. 2015). For example, homing of melanoma exosomes to sentinel lymph nodes enhances recruitment of melanoma cells, extracellular matrix deposition in the lymph nodes (Hood et al. 2011). A few studies have focused on gene expression and proteomics of PTC with lymph node metastasis (LNM) (Pagni et al. 2015, Rusinek et al. 2015, Lin et al. 2016, Mutalib et al. 2016), but they investigated thyroid lesions. How tumour exosomes mediate PTC metastasis remains an unresolved question.

Exosomes contain functional biomolecules (including proteins, RNA, DNA and lipids) that can be horizontally transferred to recipient cells with preservation of their function (Colombo et al. 2014). Considering that microarray results cannot fully represent protein changes due to post-transcriptional and posttranslational regulation, we used proteomic approaches to study the differentially expressed proteins in serumpurified exosomes (SPEs) of PTC patients. By using a combination of liquid chromatography-tandem mass spectroscopy (LC-MS/MS) analyses and tandem mass tag (TMT) label quantitation, we compared the proteomes of SPEs of PTC patients with LNM, those without LNM and healthy volunteers. In this study, we aim to identify the characteristics and biological functions of serum exosomes in lymph node metastases of PTC.

\section{Materials and methods}

\section{Patients and serum samples}

Sixteen healthy volunteers, 16 patients diagnosed with PTC with LNM and 17 patients diagnosed with PTC without LNM were recruited to this study at Peking University Cancer Hospital (Beijing, China). To better investigate the underlying molecular mechanisms of lymph node metastases of PTC patients, we endeavoured to obtain samples from PTC patients aged over 45 years, and 16 patients with LNM and 17 patients without LNM (non-LNM). Sixteen healthy volunteers were over the age of 40 years without diabetes, blood diseases or tumours. According to the WHO classification of PTC (2017 edition), we investigated papillary carcinoma and papillary microcarcinoma. The H\&E images of all of these PTC patients are shown in the Supplementary Fig. 1 (see section on supplementary data given at the end of this article). The selection criteria used in this study were strictly according to the TNM staging system recommended by the American Joint Cancer Committee (AJCC Seventh Edition). None of the patients had chemotherapy or radiotherapy before serum samples were collected. Information on all the patients was obtained from Peking University Cancer Hospital (Table 1). Informed consent was obtained from all patients. The study was performed with the approval of the Ethics Committee of Peking University Cancer Hospital. The whole blood was collected in a centrifuge tube for $2 \mathrm{~h}$ at room temperature then centrifuged at $3000 \mathrm{~g}$ for $10 \mathrm{~min}$. The supernatant was stored at $-80^{\circ} \mathrm{C}$ and each patient donated $1-1.5 \mathrm{~mL}$ serum.

\section{Bioinformatics analysis}

In this study, the proteins identified were classified by FunRich software version 3 and by Gene Ontology (GO) terms, based on the given protein's molecular function, cellular component and biological process. Enrichment analysis was performed using the Web-based Gene Set AnaLysis Toolkit (WebGestalt; Vanderbilt University, Nashville, TN, USA). Statistical significance of pathways was based on a $P$ value $<0.05$ and the presence of at least four target genes in the WikiPathway (see experimental workflow in Supplementary Fig. 2).

\section{Statistical analysis}

All replicates were true biological replicates. Unless indicated, statistical significance of differences was evaluated by one-way ANOVA test. $P$ value $<0.05$ was considered statistically significant.

\section{Reagents}

Reagents and kits were purchased from commercial sources. The Total Exosome Isolation Reagent was purchased from Invitrogen. Iodoacetamide (IAA), dithiothreitol (DTT), urea and cell culture medium were from GE Healthcare (Little Chalfont, Bucks, UK). The BCA protein assay kit, TMT Mass Tagging Kits and foetal bovine serum (FBS) were from Thermo Fisher Scientific, and protease inhibitor cocktail was from Roche. Sequencing-grade endoproteinase Trypsin/Lys-C was from Promega. Transwell Permeable Support $8.0 \mu \mathrm{m}$ Polyester (PET) Membrane $6.5 \mathrm{~mm}$ Insert and Matrigel Basement 


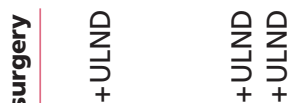

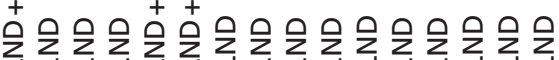

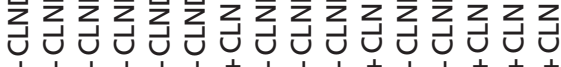

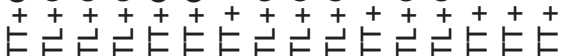

$$
\text { 可 }
$$

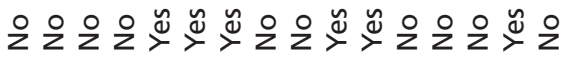

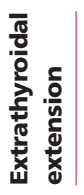

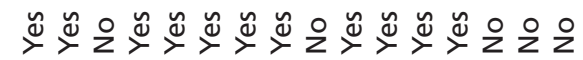

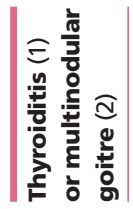

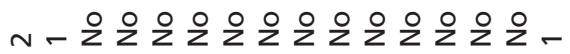

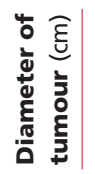

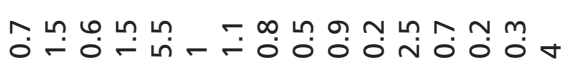

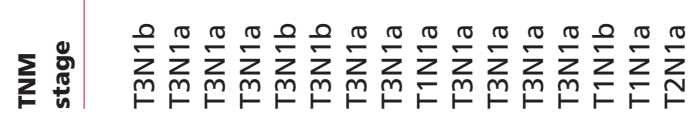

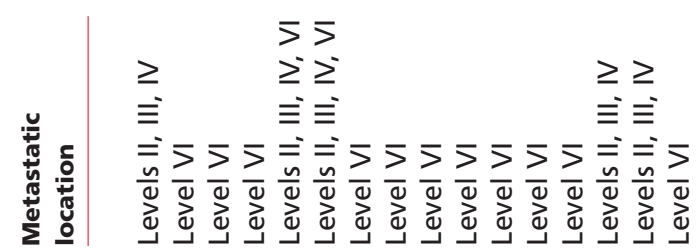

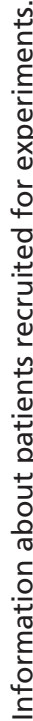



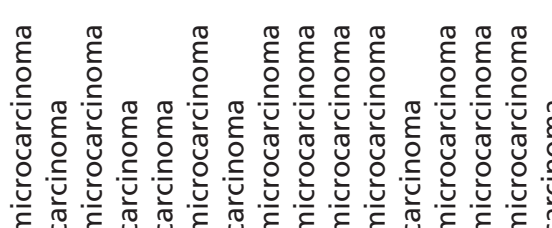

皇

응 응 응 흥 흥 응 응 흥 응 응 응 흥 응 응 흥 응

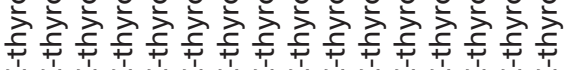

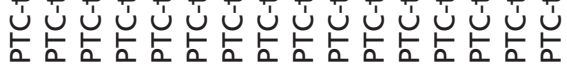

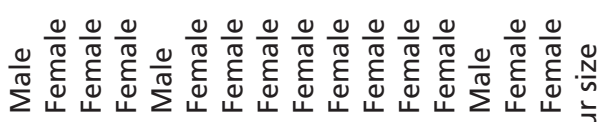

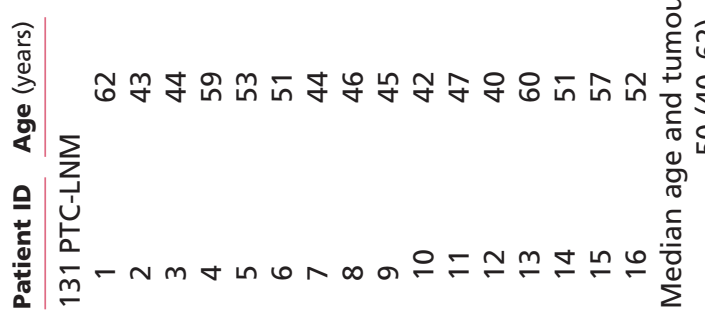

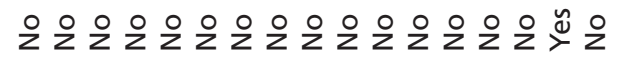

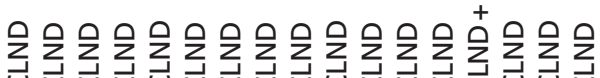

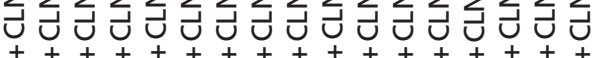

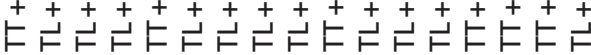

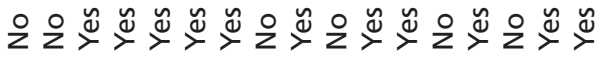

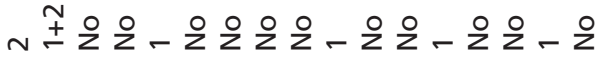

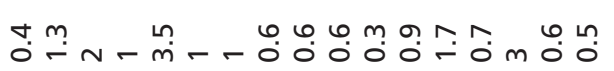

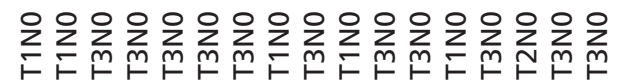

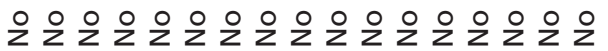

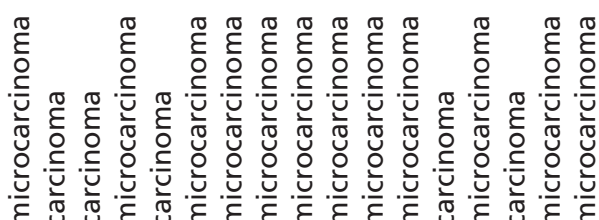

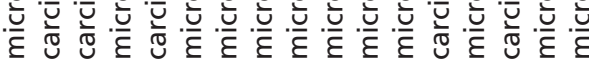

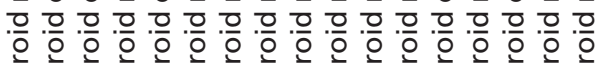

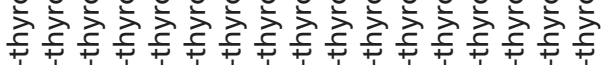

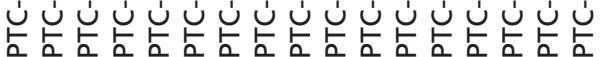

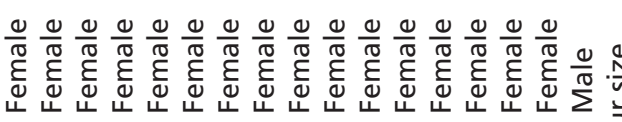

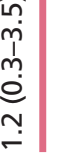

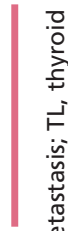

(1)

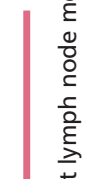

(1)

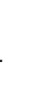


Membrane Matrix were obtained from Corning (Corning, NY, USA). An Xbridge BEH300 C18 column $(4.6 \times 250 \mathrm{~mm}$, $5 \mu \mathrm{m}$ ) was obtained from Waters (Milford, MA, USA). A fused silica capillary column ( $75 \mu \mathrm{m}$ ID, $150 \mathrm{~mm}$ length) was purchased from Upchurch (Oak Harbor, WA, USA), and $\mathrm{C} 18$ resin $(300 \AA, 5 \mu \mathrm{m})$ was from Varian (Palo Alto, CA, USA).

\section{Isolation of exosomes}

The serum samples from PTC patients with LNM, healthy volunteers and without LNM were pooled together, respectively, to perform isolation of exosomes by ultracentrifugation and total exosome isolation reagent. For ultracentrifugation, the supernatant was first centrifuged at $10,000 \mathrm{~g}$ for $30 \mathrm{~min}$ at $4^{\circ} \mathrm{C}$. The supernatant was then transferred into ultracentrifuge (UC) tubes with total volume of $18.5 \mathrm{~mL}$. UC was performed using a Beckman Optima L-100XP Ultracentrifuge at $110,000 \boldsymbol{g}$ at $4^{\circ} \mathrm{C}$ for $90 \mathrm{~min}$. After the UC step, the supernatant was removed, followed by addition of $1 \mathrm{~mL}$ PBS to wash the pellet without disturbing it. The pellet was then dissolved with $40 \mu \mathrm{L} 8 \mathrm{M}$ urea after the PBS was removed. The total protein concentration was determined using a NanoDrop 2000 spectrophotometer (Thermo Scientific). For the isolation of exosomes by total exosome isolation reagent, the required volume of pooled serum sample was first diluted with an equal volume of PBS to decrease viscosity, and then 0.2 volumes of the total exosome isolation reagent were added. The serum/reagent mixture was mixed by vortexing until it was homogenous, then it was incubated at $4^{\circ} \mathrm{C}$ for $30 \mathrm{~min}$. Next, the sample was centrifuged at $10,000 \boldsymbol{g}$ for $10 \mathrm{~min}$ at room temperature and the supernatant was discarded. The pellet from every $100 \mu \mathrm{L}$ serum was resuspended in $25 \mu \mathrm{L}$ PBS for Western blotting and Transwell assays. A fraction of the resuspended isolated exosomes was lysed with RIPA buffer and then the protein concentration was determined using a BCA protein assay kit.

\section{TMT labelling}

For proteolytic digestion, $100 \mu \mathrm{g}$ protein of the lysate in $8 \mathrm{M}$ urea were alkylated with DTT and IAA. Digestion with trypsin/Lys-C followed the manufacturer's protocol and the reaction was quenched by heating at $60^{\circ} \mathrm{C}$. Digested proteins were desalted, dried and finally dissolved in $200 \mathrm{mM}$ triethylammonium bicarbonate buffer $(\mathrm{pH}=8.5)$. TMT labelling was performed using the TMT Kit following the manufacturer's protocol. Different TMT labels were used to label the different groups: TMT-129 was used for the healthy volunteers; TMT-130 was used for the PTC patient without LNM group; TMT-131 for the PTC patient with LNM group. After labelling, samples were pooled, dried and dissolved in $0.1 \%$ trifluoroacetic acid (TFA). The dissolved samples were desalted and dried again, and finally dissolved in $100 \mu \mathrm{L}$ of $0.1 \%$ TFA.

\section{High-performance liquid chromatography analysis}

The TMT labelled peptides $(100 \mu \mathrm{L}$ in $0.1 \%$ TFA) were transferred to tubes for high-performance liquid chromatography (HPLC) (UltiMate 3000 UHPLC, Thermo Scientific) fractionation, using an Xbridge BEH300 $\mathrm{C} 18$ column maintained at $45^{\circ} \mathrm{C}$ with a flow rate of $1.0 \mathrm{~mL} / \mathrm{min}$. UV absorbance was detected at $214 \mathrm{~nm}$. The gradient elution buffer consisted of $\mathrm{ddH}_{2} \mathrm{O}$ (phase A, $\mathrm{pH}$ 10.0) and $98 \%$ acetonitrile (phase $\mathrm{C}, \mathrm{pH}$ 10.0). Each $1.5 \mathrm{~mL}$ fractions were collected into a microcentrifuge tube, and then dried. Samples were combined according to the peptide abundance and dissolved in $20 \mu \mathrm{L}$ of $0.1 \%$ TFA for liquid chromatography LC-MS/MS analysis.

\section{Peptide analysis by LC-MS/MS}

Briefly, following separation using a 135-min gradient elution at a flow rate of $0.3 \mu \mathrm{L} / \mathrm{min}$ in the Ultimate U3000 system, the protein digests were analysed with a directly interfaced Thermo Orbitrap Fusion Lumos mass spectrometer. The analytical column consisted of a homemade fused silica capillary column $(75 \mu \mathrm{m}$ ID, $150 \mathrm{~mm}$ length; Upchurch) packed with C-18 resin ( $300 \mathrm{~A}, 2 \mu \mathrm{m}$; Varian). Mobile phase A consisted of $0.1 \%$ formic acid, and mobile phase B consisted of 100\% acetonitrile and $0.1 \%$ formic acid. The Orbitrap Fusion mass spectrometer was operated in the data-dependent acquisition mode using Xcalibur 4.1 software. A single full-scan mass spectrum was obtained in the Orbitrap (350-1550 m/z, 120,000 resolution) followed by 3-s datadependent MS/MS scans in an Ion Routing Multipole at $35 \%$ normalized collision energy (HCD).

\section{Data analysis}

Protein identification were analysed using Proteome Discoverer 2.1 software (Thermo Scientific) with the SEQUEST search engine. In detail, the raw MS data files were analysed against the UniProt/SwissProt human proteome database (20,238 items, released on 2016.09.07). The searching criteria were set as the following parameters: 
precursor mass tolerance, $5 \mathrm{ppm}$; fragment mass tolerance, $0.02 \mathrm{Da}$; total intensity threshold, 20,000; minimum number of peaks, 200; a maximum of two missed cleavages was allowed. Carbamidomethylation (on C) and TMT 6-plex (on $\mathrm{K}$ and the peptide $\mathrm{N}$-terminus) were set as static modifications, and oxidation (methionine, M) was specified as a dynamic modification. Protein identification was considered valid if at least one peptide was statistically significant $(P<0.05$ with a false discovery rate at $1 \%)$. Protein quantification was performed using the TMT 6-plex method. Reporter monoisotopic m/z was tuned according to the raw spectral data. Proteins were quantified with unique peptides. Representative MS/MS spectral data of identified peptides and the intensity of TMT precursor ions were used for protein quantification.

\section{Western blot}

Protein concentration was determined using a $\mathrm{BCA}$ kit (Thermo Scientific). The SPEs sample proteins were separated by $10 \%$ SDS-PAGE and transferred onto PVDF membranes. Proteins were then subjected to immunoblot. All primary and secondary antibodies were diluted in PBS. Primary antibody incubation was performed at $4^{\circ} \mathrm{C}$ overnight, and secondary antibody incubation was performed at room temperature for $2 \mathrm{~h}$. The immunoreactive blots were visualized using an enhanced chemiluminescence reagent. Antibodies against CAPNS1 (rabbit monoclonal, 1/2000, ab92333), TLN1 (rabbit polyclonal, 1/400, ab71333) was purchased from Abcam (Cambridge, Cambs, UK). Antibodies against SRC (rabbit polyclonal, 1/1000, CST2108), ALDOA (rabbit polyclonal, 1/1000, 3188S) and ITGB2 (rabbit polyclonal, 1/1000, CST4702) were purchased from Cell Signaling Technology. Cd63 (rabbit polyclonal, 1/200, sc-15363), Annexin1 (mouse monoclonal, 1/200, sc-7964), HSP27 (mouse monoclonal, 1/5000, sc-13132) were purchased from Santa Cruz Biotechnology. We used Coomassie Brilliant Blue staining as a loading control. Densitometric analysis was performed on the Western blot images using ImageJ software (nih.gov).

\section{Immunohistochemistry}

Tissue sections were deparaffinized in xylene and hydrated in ethanol (EtOH) using standard histological procedures. Sections were then subjected to a 30-min heat-mediated antigen retrieval step using a sodium citrate buffer (sodium citrate $10 \mathrm{mM}, 0.05 \%$ Tween 20, pH 6.0). Endogenous peroxidase activity was blocked using the 3\%
$\mathrm{H}_{2} \mathrm{O}_{2}$ blocking solution before sections were incubated with primary antibodies. Incubation with secondary antibodies and detection was performed using the ZSGB-BIO DAB Detection kit.

\section{Cell invasion and migration assay}

Cell invasion assays were performed using Transwell Permeable Support $8.0 \mu \mathrm{m}$ Polyester (PET) Membrane $6.5 \mathrm{~mm}$ Inserts ( $8 \mathrm{~mm}, 24$-well format), which were coated with $10 \mu \mathrm{g}$ diluted Matrigel Basement Membrane Matrix. Assays were performed as follows: the cells were seeded into the upper chamber at $3 \times 10^{4}$ per insert in $100 \mu \mathrm{L}$ serum-free medium, and then complete medium with $10 \%$ FBS was added outside of the insert as a chemoattractant. Briefly, the exosomes isolated from the serum of PTC patients or normal volunteers were added to the top of the Transwell insert; an equal volume of exosome-free PBS was added as the blank control. The chamber was cultured in a $37^{\circ} \mathrm{C}, 5 \%$ $\mathrm{CO}_{2}$ incubator for $24 \mathrm{~h}$. Then, cells were removed from the upper chamber, and the chambers were fixed with methanol for $30 \mathrm{~min}$. Cells outside the insert membrane were stained with $0.2 \%$ crystal violet for $50 \mathrm{~min}$. The number of cells on the lower surface of the membrane was counted in five different fields. Three independent experiments were performed with triplicate wells. The protocol used for the migration assay was the same as that used for the invasion assay, except that the Transwell insert was not coated with Matrigel, and the cells were seeded at $5 \times 10^{4}$ per insert. Twice biological replicates were included.

\section{Results}

\section{Proteomic profiles of PTC with LNM and PTC without LNM show marked differences in protein expression}

The proteomics mass spectroscopy raw data from this study has been deposited to the ProteomeXchange Consortium via the PRIDE partner repository, with dataset identifier PXD005916.

Using LC-MS/MS analysis, a total of 1569 proteins were identified by two or more unique peptides (Supplementary Table 1). Based on the expression threshold given in 'Data analysis', differentially expressed proteins (DEPs) were filtrated (LNM/non-LNM protein abundance ratio $\geq 1.2$ or $\leq 0.8$ ). The accession numbers, gene names, score, unique peptides and relative ratios of the DEPs are listed in Supplementary Table 2. Pairwise comparisons using SPEs from normal volunteers as a 
control group showed the profile of molecular changes in PTC-NLNM patients was different from that in PTC-LNM patients (Fig. 1). We found that the protein expression of PTC-LNM group exhibited marked increases compared to the PTC-NLNM and normal groups in cluster 3 of the heatmap (original quantitative data for the heatmap can be found in Supplementary Table 3).

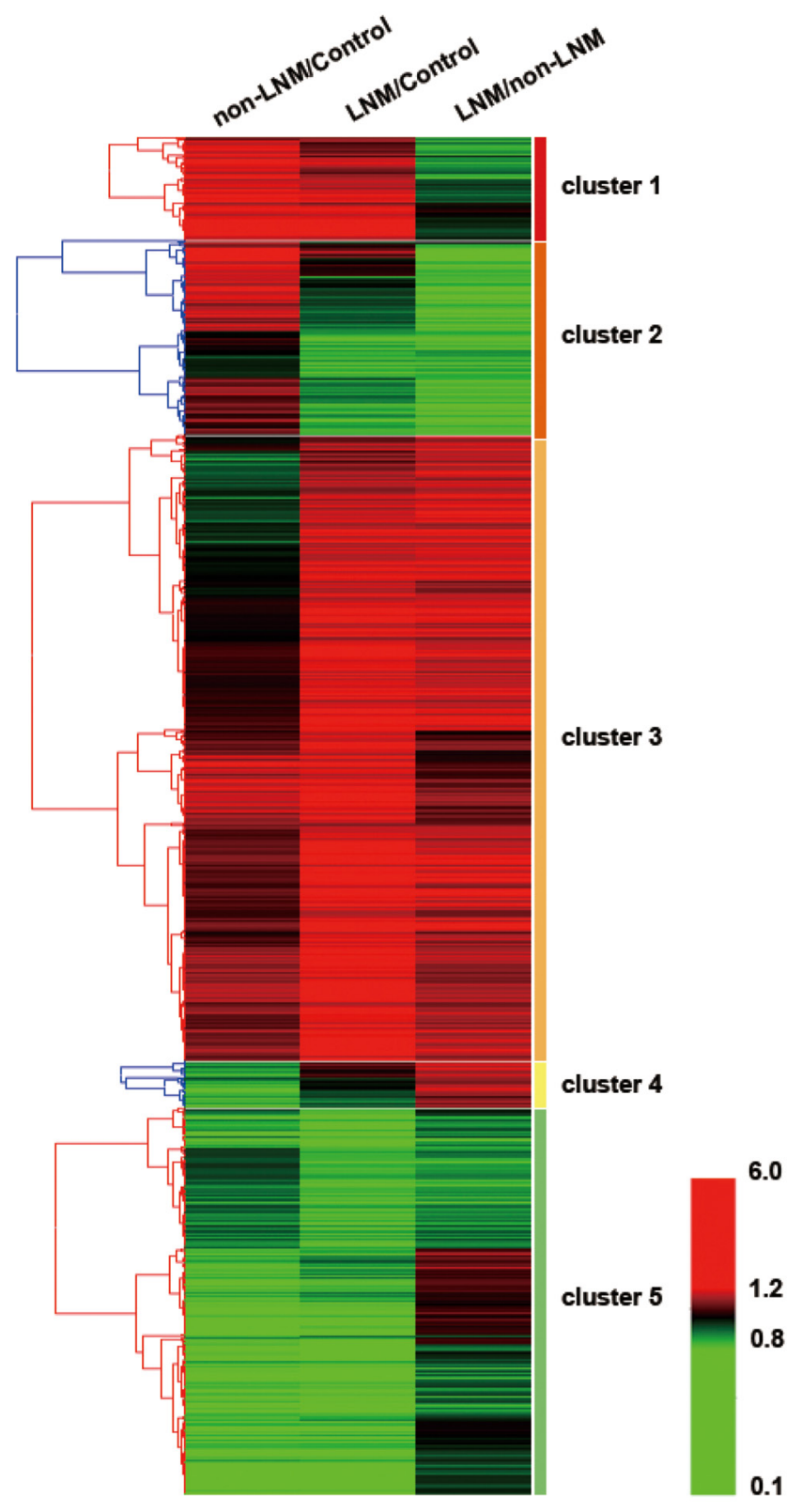

Figure 1

Hierarchical clustering of papillary thyroid cancer (PTC) vs normal specimens, and PTC with lymph node metastasis (LNM) vs PTC without LNM (non-LNM), using Heml software (Heatmap Illustrator, version 1.0). Red and green indicate upregulation or downregulation, respectively. A full colour version of this figure is available at https://doi.org/10.1530/ ERC-17-0547.

\section{GO and molecular pathways of DEPs}

To identify PTC with LNM-related physiological processes implicated by the exosomal proteins, we clustered DEPs into GO categories using bioinformatics tools. Most of the SPEs proteins in the PTC with LNM group in cluster 3 (Fig. 1) were elevated compared with the control and non-LNM groups. So, we focused on the DEPs in cluster 3. When referring to the GO category 'cellular component' (Fig. 2A), the DEPs were most enriched in the categories cytoplasm and exosomes. In terms of 'biological process' classification, the DEPs were involved in signal transduction and cell communication (Fig. 2B). In the context of 'molecular function' classification, GTPase activity and cytoskeletal protein binding were the major functional classes of the DEPs (Fig. 2C).

To gain further insight, we performed analysis using Cytoscape (3.4.0) software based on WikiPathways and the STRING (Protein-Protein Interaction Networks) database. Nearly all the proteins involved in the integrin-mediated cell adhesion pathway were upregulated in the PTC with LNM group compared with the PTC without LNM group (Fig. 3A). By using the Web-based GEne SeT Analysis Toolkit, we found that 20 proteins were enriched in the category neoplasm invasiveness (Supplementary Table 4) and 22 proteins were enriched in the category neoplasm metastasis (Supplementary Table 5). To understand their protein-protein interactions, we constructed protein networks using STRING. There were 20 and 19 proteins, which were mapped in STRING networks for neoplasm invasiveness and neoplasm metastasis, respectively (Fig. 3B and C). All proteins were mapped in the pathway, protein network and represented with the same series but with different saturations according to their expression level.

\section{Extraction efficiency of SPEs and verification of protein expression levels by Western blotting and immunohistochemistry}

The pooled serum samples of three PTC-NLNM patients, three PTC-LNM patients and three normal volunteers were independently subjected to isolation of exosomes using total exosome isolation reagent. Then, we assessed extraction efficiency of the SPEs. The exosome markers CD63 and Annexin 1 were validated by Western blot analysis (Supplementary Fig. 3A). Coomassie Brilliant Blue staining was used as a loading control (Supplementary Fig. 3B). In addition, we performed Western blot assays to verify the results from quantitative proteomics. Because the bioinformatics analysis found that the 
A Cellular component

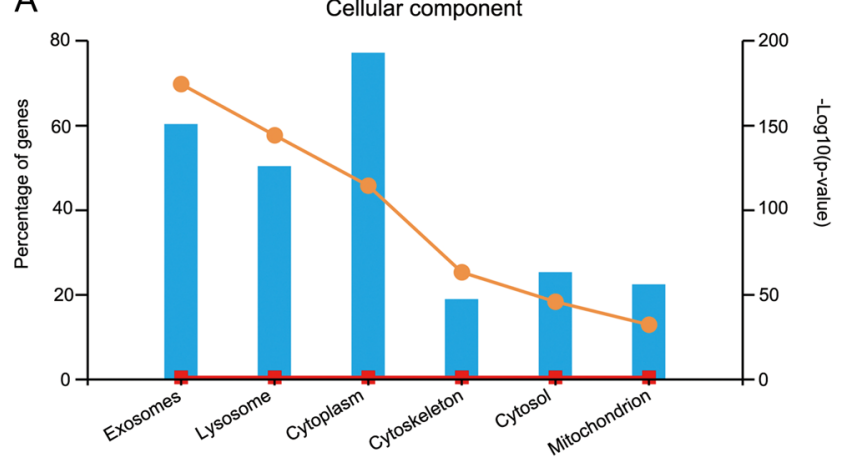

B

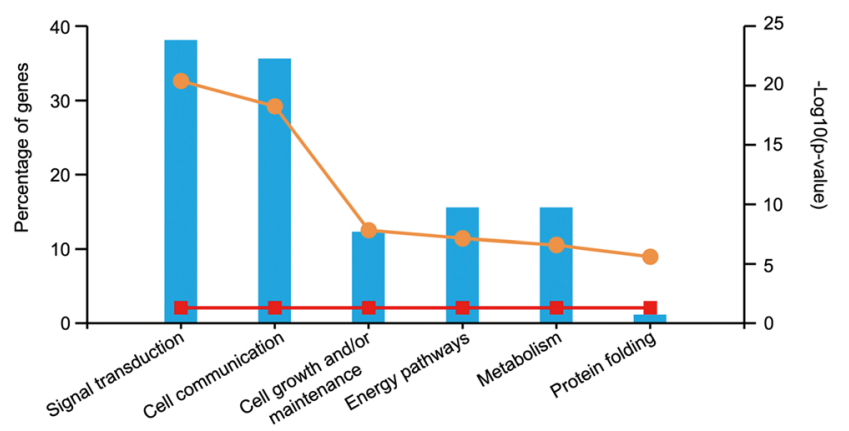

C

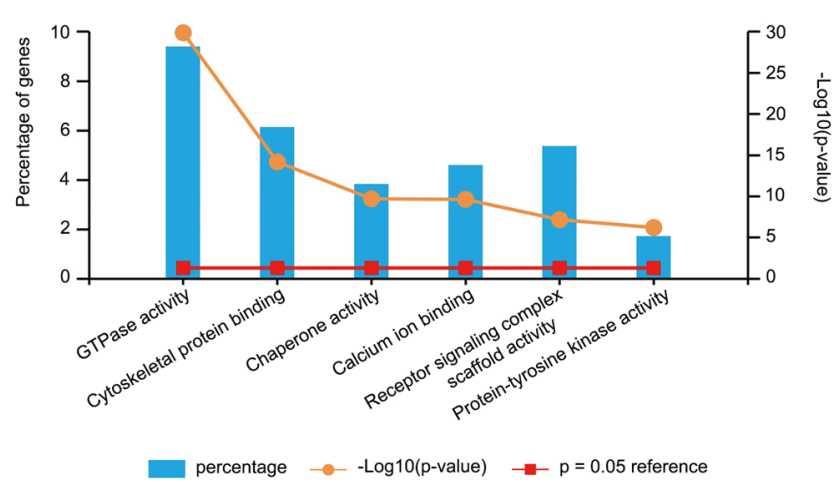

Figure 2

Characterization of identified proteins in cluster 3 of the heatmap shown in Fig. 1. The percentages are of all identified proteins in cluster 3 in the Gene Ontology categories (A) cellular component, (B) biological process and (C) molecular function. A full colour version of this figure is available at https://doi.org/10.1530/ERC-17-0547.

differential expression proteins, which are from SPEs of PTC patient with LNM compared without LNM were enriched in integrin-mediated cell adhesion pathway. Moreover, TLN1, ITGB2, SRC and CAPNS1 and are at upstream of the integrin pathway and these proteins are significantly highly expressed in serum exosomes of patients with thyroid cancer lymph node metastases, so we selected the four proteins (TLN1, ITGB2, SRC and CAPNS1) involved in the integrin signalling pathway and another four proteins (ACTB, ANXA1, HSP27, ALDOA) for Western blotting (Fig. 4). Coomassie Brilliant Blue staining was used as a loading control (Supplementary Fig. 3C). The expression levels of these DEPs showed a consistent trend with our mass spectrometry results in the SPEs of individual healthy volunteers, PTC-NLNM and PTC-LNM patients. However, due to the limited number of samples and the differences between samples, none of these DEPs were shown to be differentially expressed in a statistically significant manner by densitometric analysis. Furthermore, we performed immunohistochemistry assays to confirm the mass spectrometry results of TLN1, SRC, CAPNS1, ACTB, ANXA1, HSP27 and ALDOA by using paraffin-embedded tissue sections from another 14 PTC-NLNM patients and 14 PTC-LNM patients (Fig. 5). Detailed PTC patient information validated by immunohistochemistry is shown in the Supplementary Table 6. In addition, we retrieved mRNA sequencing gene expression data of 209 thyroid carcinoma patients including clinical information from The Cancer Genome Atlas dataset (TCGA, Provisional) through the TCGAAssembler 2 software. We found that mRNA expression levels were upregulated in the PTC-LNM patients tumourous tissues compared with the PTC-NLNM patients tumourous tissues, including SRC $(P=0.004)$, ACTB $(P=0.009)$ and ANXA1 $(P=0.007)$. However, TLN1, ITGB2, CAPNS1, ALDOA, HSPB1 were not shown to be differentially expressed in a statistically significant manner (Supplementary Fig. 4). Detailed TCGA dataset information is shown in the Supplementary Table 7.

\section{PTC-derived SPEs promote cellular invasion}

We performed Transwell assays to demonstrate the effects of SPEs on the invasion and migration properties of two thyroid cancer cell lines, BCPAP and BHT101. Addition of $30 \mu \mathrm{g}$ or $100 \mu \mathrm{g}$ SPEs from PTC patients with LNM significantly increased the invasion ability of BHT101 cells. The migration ability of BHT101 cells did not show significant alternation. However, the invasion and migration ability of BCPAP cells were not obviously affected by the presence of SPEs from PTC patients with LNM compared with SPEs from PTC patients without LNM (Fig. 6).

\section{Discussion}

This is the first global proteomic analysis that compares the SPEs protein signatures of PTC with those of healthy volunteers. Our proteomic analysis is based on pooled samples and does not display the inter-patient variability 

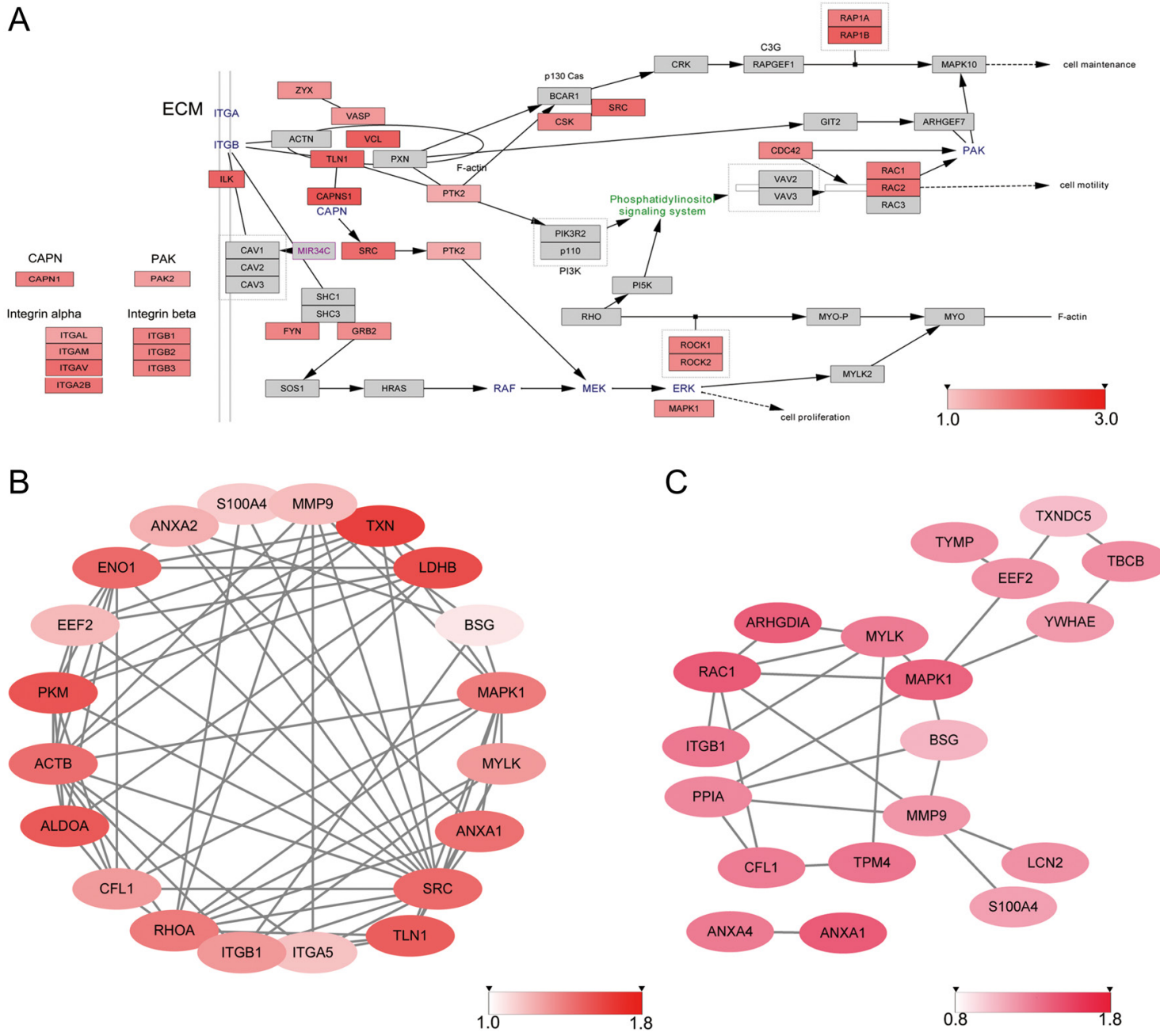

Figure 3

Pathway and interaction network analysis of proteins with altered expression in a comparison of PTC-LNM with PTC without LNM (NLNM). The relative protein expression level in serum-purified exosomes (SPEs) is indicated by different shading. Proteins in grey were not identified in this study. (A) Integrinmediated cell adhesion pathway; (B) interaction network of proteins related to GEne SeT Analysis Toolkit category neoplasm invasiveness; (C) interaction network of proteins related to category neoplasm metastasis. A full colour version of this figure is available at https://doi.org/10.1530/ERC-17-0547.

A

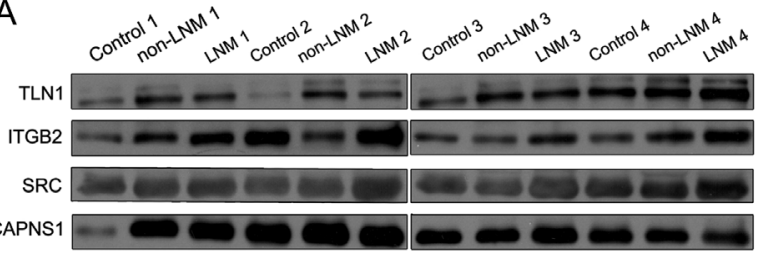

B

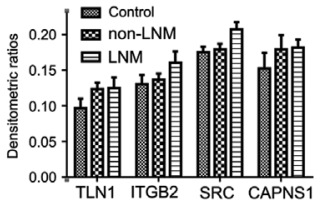

D

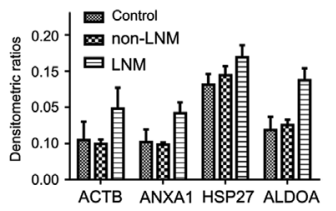

Figure 4

Western blot validation of differentially expressed proteins (DEPs) identified by mass spectrometry. (A and $C$ ) Individual validation of TLN1, ITGB2, SRC, CAPNS1, ACTB, ANXA1, HSP27 and ALDOA. 'Control 1-4', 'non-LNM 1-4' and 'LNM 1-4' represent the SPEs of individual healthy volunteers, PTC patients without LNM, and PTC patients with LNM, respectively. (B and D) The optical density of each immunoreactive band was normalized with total loaded proteins (Coomassie stain). The densitometric ratios are shown in the histogram. 

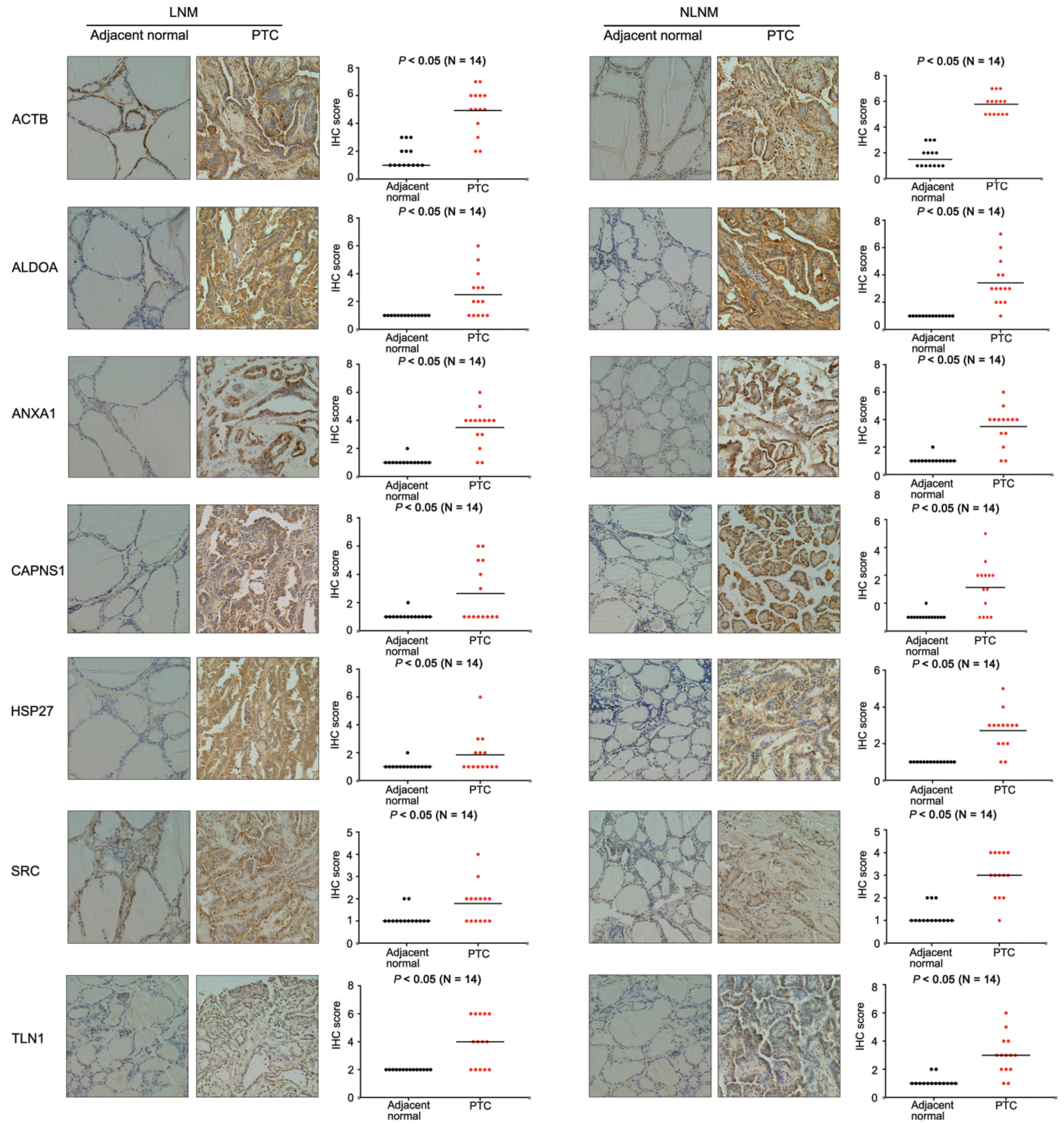

\section{Figure 5}

Representative images and IHC scores of differentially expressed proteins (DEPS) in an additional 14 PTC patients with LNM and 14 PTC patients without LNM. Significance was determined using Student's $t$ test. A full colour version of this figure is available at https://doi.org/10.1530/ERC-17-0547.

that would be seen from analysis of individual samples. In this study, comparison of SPEs from PTC patients with LNM to those without LNM by TMT-based MS/MS yielded a large number of differentially expressed proteins (DEPs), revealing obvious differences in protein signals connected with tumour metastasis.

http://erc.endocrinology-journals.org https://doi.org/10.1530/ERC-17-0547

\section{SPEs of PTC patients with LNM as mediators of invasion and migration}

In the network of 20 proteins related to neoplasm invasiveness, the proteins SRC and ACTB serve as hubs at which protein interactions converge. The nonreceptor 

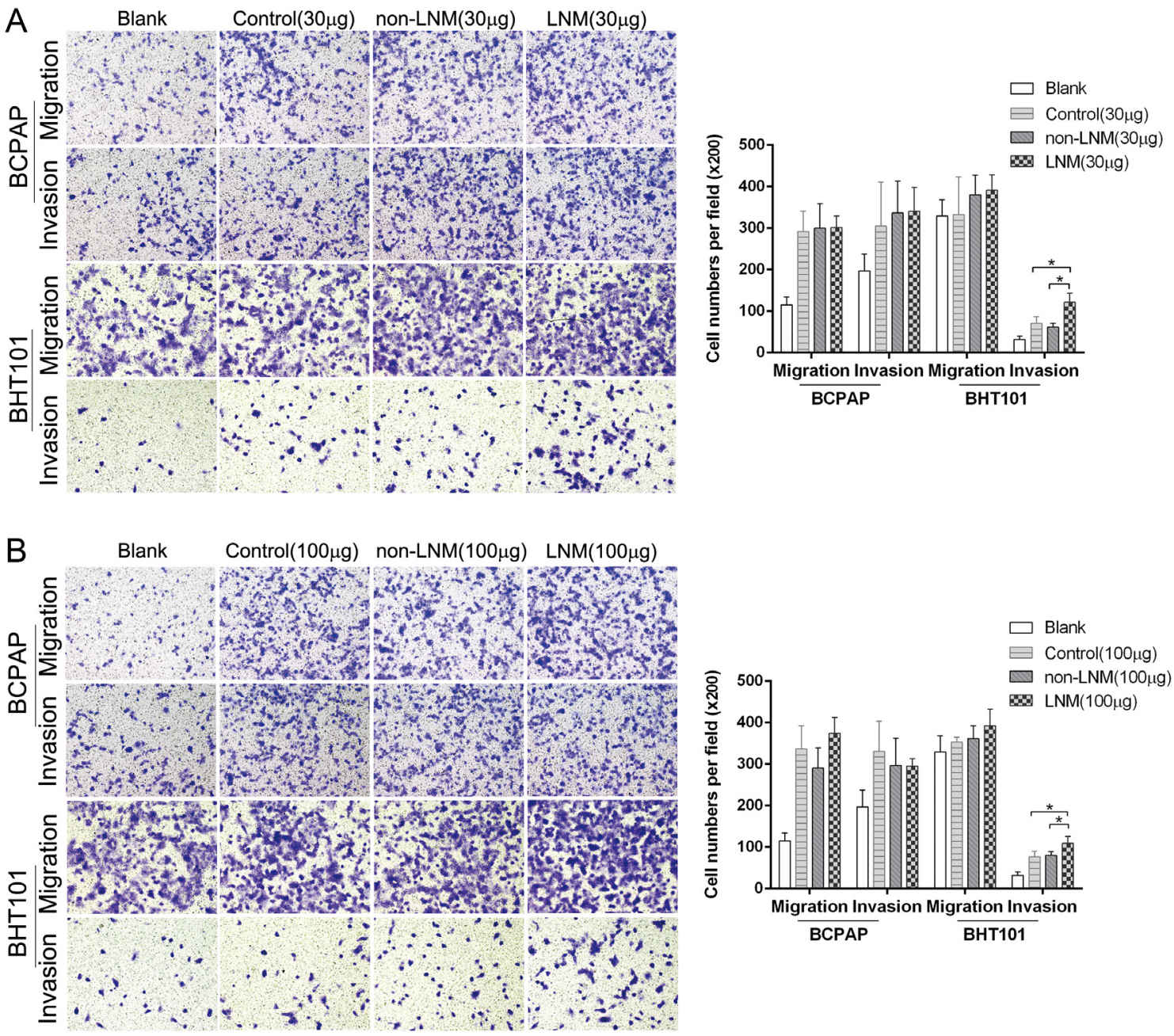

Figure 6

SPEs derived from PTC patients with LNM promote PTC cell invasion. Transwell assays were used to determine the invasion and migration of BCPAP and BHT101 cells treated with SPEs from PTC patients with LNM, PTC patients without LNM, or SPEs from normal controls. PBS was used as a blank.

(A) Treatment of BCPAP and BHT101 cells with $30 \mu \mathrm{g}$ SPEs; (B) $100 \mu \mathrm{g}$ SPEs. A full colour version of this figure is available at https://doi.org/10.1530/ ERC-17-0547.

protein tyrosine kinase SRC and its family members have been found to be elevated in multiple cancers (Summy \& Gallick 2003). Similarly, our experiment firstly observed that SRC was upregulated in serum exosome from PTC patients with LNM. SRC can regulate protumourigenic functions via activation of downstream signalling pathways, including the extracellular signal-regulated kinase (MAPK/ERK) and focal adhesion kinase (FAK) pathways (Chan et al. 2012). SRC inhibitors have been reported to decrease PTC cell invasion and proliferation (Henderson et al. 2014). Further, in orthotopic nude mouse models, dasatinib, which inhibits SRC activity, could effectively slow the development of lymph node metastases in tumours (Park et al. 2008). We believe that
SRC plays a central role in exosome-mediated metastasis of PTC and implies as a potential target for cure PTC.

ACTB is usually regarded as a housekeeping gene. However, accumulating evidence suggests that ACTB is closely related to a variety of cancers (Guo et al. 2013). In our results, АСТВ was obviously upregulated when the SPEs of PTC patients with LNM compared to those without LNM. Abnormal expression of ACTB and the resulting changes to the cytoskeleton might enhance cancer cell motility and are associated with the invasiveness and metastasis of cancers (Guo et al. 2013). Additionally, the glycolysis-related protein fructosebisphosphate aldolase A (ALDOA) increased sharply in the serum exosome of PTC patients with LNM. ALDOA http://erc.endocrinology-journals.org https://doi.org/10.1530/ERC-17-0547
(2) 2018 Society for Endocrinology Published by Bioscientifica Ltd. Printed in Great Britain 
has been found to correlate with pancreatic cancer cell metastasis capacity (Ji et al. 2016). ALDOA also provided good survival prediction for TNM stage I-IV colorectal cancer patients (Peng et al. 2012). Our result of Western blot has revealed that the expression of ALDOA was gradually increased with the progression of PTC. In the network of 19 DEPs related to neoplasm metastasis, MAPK1 and RAC1 serve as hubs at which protein interactions converge. In PTC patients with LNM group, MAPK1 and RAC1 were also aberrantly overexpressed compared with PTC patients without LNM group. MAPK1 is also known as ERK2. Upregulation of the ERK pathway has been shown to contribute to tumour invasion and progression (von Thun et al. 2012). Activation of RAC1 induces membrane protrusion and is associated with increased focal adhesion (Zhen et al. 2013). It has been confirmed that the knockdown of RAC1 reduced cell migration and invasion in TPC-1 and HTH83 thyroid cancer cells (Wang et al. 2015). Our Transwell experiment has revealed that the exosome from PTC patients with LNM is able to improve the invasion of BHT101 thyroid cancer cells. Consequently, it is possible that exosomal RAC1 is a key modulator of migration and invasion of thyroid cancer cells.

Transwell assays showed that SPEs from PTC patients with LNM were able to promote the invasion ability of BHT101 cells, but not BCPAP cells. We suppose that this may be because BCPAP is a differentiated thyroid carcinoma (DTC) cell line (Fabien et al. 1994), and DTC shows poor invasion potential (Dralle \& Machens 2008). However, BHT-101 was established in vitro from a metastatic lymph node deposit in a female patient with anaplastic PTC (Pályi et al. 1993). The BHT-101 cell line is more aggressive than the BCPAP cell line. According to our data, SPEs from PTC patients with LNM had weak influence on the migration ability of thyroid cancer cells. Therefore, we suggest that the function of SPEs is very complex, and there is a serious limitation on imitating interplay between SPEs and recipient cells in in vitro systems.

\section{SPEs in PTC patients with LNM play critical roles in epithelial-to-mesenchymal transition}

The early events of metastatic dissemination are thought to be initiated by epithelial-to-mesenchymal transition (EMT) processes in carcinoma cells (Gao et al. 2012). To gain insights into the role of SPEs in EMT, we compared our data with several studies that investigated key factors that modulate the progression of EMT. Several mediators of EMT were identified in the present study, including ANXA1 and HSP27. ANXA1 is a calcium/phospholipidbinding and actin regulatory protein (Lim \& Pervaiz 2007) that can enhance TGF $\beta /$ Smad signalling and actin reorganization (Graauw \& Thompson 2010). TGF $\beta /$ Smad signalling is a major driving factor of EMT (Polyak \& Weinberg 2009). ANXA1 has been found to regulate an EMT-like phenotypic switch and promote metastasis (Graauw \& Thompson 2010). Equally important, it has been reported that ANXA1 may help to distinguish benign vs malignant thyroid nodules (Ciregia et al. 2016). Our data provide evidence that ANXA1 represents a potential biomarker of thyroid cancer. Mass spectrum analysis of SPEs of pooled serum from PTC patients with LNM demonstrated that ANXA1 was significantly upregulated in these patients. The aberrant expression of ANXA1 was validated by performing Western blot analysis of individual SPE samples. We reasoned that exosomal ANXA1 improves EMT and metastatic potential in PTC, but this requires further research in vitro and in vivo. HSP27 is an ATPindependent molecular chaperone and has been identified as a critical mediator in cancer progression (Rocchi et al. 2005). Recently, HSP27 was shown to be a marker of tumour cells associated with lymphatic metastasis of breast cancer (Kaigorodova et al. 2015). In contrast, HSP27 has not been reported to be related to lymphatic metastasis of PTC. Our data show that HSP27 was aberrantly overexpressed in SPEs of PTC patients with LNM, raising the possibility that HSP27 is a predictive marker of lymphatic metastasis of PTC. From the above findings, we propose that SPEs of PTC patients with LNM mediate EMT and promote metastatic dissemination.

\section{Integrin signal pathway plays key roles in exosome-mediated metastasis}

Integrins are a family of heterodimeric cell surface receptors that control cell adhesion, migration, proliferation and survival by forming a physical connection between the cell and the extracellular matrix (Desgrosellier \& Cheresh 2010). Previous study has shown that tumour-derived exosomes taken up by organ-specific cells prepared the premetastatic niche, and exosomal integrin expression patterns were associated with metastatic organotropism (Hoshino et al. 2015). Consistent with this, our analysis in this study shows that several of the DEPs are typical proteins implicated in the integrin-mediated cell adhesion 
pathway: ITGA2, ITGA2B, ITGAV, ITGB1, ITGB2 and ITGB3 are overexpressed in SPEs from PTC patients with LNM relative to those from PTC patients without LNM. Moreover, we selected ITGB2, TLN1, CAPNS1 and SRC, which are upstream of the integrin pathway, to perform Western blot assays that validated the mass spectrometry results. We propose that the aberrant expression of integrins in SPEs from PTC patients with LNM promotes the formation of premetastatic niches.

In addition, we found that downstream proteins of integrins in the integrin-mediated cell adhesion pathway were significantly upregulated. TLN1 is a macromolecular cytoskeletal protein. TLN1 regulates focal adhesion signalling by conjugating integrins to the cytoskeleton and is essential for integrin activation (Desiniotis \& Kyprianou 2011). It has been reported that high expression of TLN1 increased prostate cancer metastasis to lymph nodes and bone (Jin et al. 2015). Other studies reported that TLN1 is a potential marker for early-stage diagnosis of cancer, because its aberrant expression in serum from cancer patients was sufficient to distinguish them from healthy people (Chen et al. 2017). TLN1 levels, the tumour grade and LNM are significantly correlated (Bostanci et al. 2014). This is consistent with our results. Calpains (CAPN) are a family of $\mathrm{Ca}^{2+}$-dependent cysteine proteases that are involved in cell proliferation, cell migration and invasion (Yang et al. 2017). CAPNS1 has been identified as a major protein that promotes metastasis of hepatocellular carcinoma (Dai et al. 2014). However, the involvement of CAPNS1 in the metastasis of PTC has not been reported. Our study indicates that CAPNS1 is associated with LNM of PTC, but its role in regulation of the proliferation and migration of PTC cells needs to be investigated further. SRC is a downstream protein of TLN1 and CAPNS1 in the integrin-mediated cell adhesion pathway; its functions have been discussed above. From these findings, we speculate that the elevated expression of these integrin-related proteins in SPEs of PTC patients with LNM is able to active the integrin signalling pathway in target cells. This will contribute to the formation of metastatic niches.

Although the functions of tumour-derived exosomes need to be further elucidated, the current study provides important insights into their mechanisms through detection of the profile of SPEs from PTC patients with LNM and presents a rationale for targeting certain exosomal proteins in the design of diagnostic and therapeutic methods for PTC with LNM.

\section{Supplementary data}

This is linked to the online version of the paper at https://doi.org/10.1530/ ERC-17-0547.

\section{Declaration of interest}

The authors declare that there is no conflict of interest that could be perceived as prejudicing the impartiality of the research reported.

\section{Funding}

Wei Ge is supported by the CAMS Initiative for Innovative Medicine (2017I2M-3-001), the National Science Foundation of China (No. 81572625) and Tianxiao Wang is supported by the Beijing Municipal Administration of Hospital Youth Program (No. QML20161103).

\section{References}

Beasley NJ, Lee J, Eski S, Walfish P, Witterick I \& Freeman JL 2002 Impact of nodal metastases on prognosis in patients with welldifferentiated thyroid cancer. Archives of Otolaryngology: Head and Neck Surgery 128 825-828. (https://doi.org/10.1001/ archotol.128.7.825)

Bostanci O, Kemik O, Kemik A, Battal M, Demir U, Purisa S \& Mihmanli M 2014 A novel screening test for colon cancer: Talin-1. European Review for Medical and Pharmacological Sciences 182533.

Cabanillas ME, Mcfadden DG \& Durante C 2016 Thyroid cancer. Lancet 388 2783. (https://doi.org/10.1016/S0140-6736(16)30172-6)

Chan CM, Jing X, Pike LA, Zhou Q, Lim DJ, Sams SB, Lund GS, Sharma V, Haugen BR \& Schweppe RE 2012 Targeted inhibition of Src kinase with dasatinib blocks thyroid cancer growth and metastasis. Clinical Cancer Research 18 3580. (https://doi. org/10.1158/1078-0432.CCR-11-3359)

Chen P, Zheng X, Zhou Y, Xu Y, Zhu L \& Qian Y 2017 Talin-1 interaction network promotes hepatocellular carcinoma progression. Oncotarget 8 13003-13014. (https://10.18632/oncotarget.14674)

Ciregia F, Giusti L, Molinaro A, Niccolai F, Mazzoni MR, Rago T, Tonacchera M, Vitti P, Giannaccini G \& Lucacchini A 2016 Proteomic analysis of fine-needle aspiration in differential diagnosis of thyroid nodules. Translational Research 176 81-94. (https://doi. org/10.1016/j.trsl.2016.04.004)

Colombo M, Raposo G \& Théry C 2014 Biogenesis, secretion, and intercellular interactions of exosomes and other extracellular vesicles. Annual Review of Cell and Developmental Biology 30255. (https://10.1146/annurev-cellbio-101512-122326)

Dai Z, Zhou SL, Zhou ZJ, Bai DS, Xu XY, Fu XT, Chen Q, Zhao YM Zhu K \& Yu L 2014 Capn4 contributes to tumour growth and metastasis of hepatocellular carcinoma by activation of the FAK-Src signalling pathways. Journal of Pathology 234 316-328. (https://doi. org/10.1002/path.4395)

Desgrosellier JS \& Cheresh DA 2010 Integrins in cancer: biological implications and therapeutic opportunities. Nature Reviews Cancer 10 9. (https://doi.org/10.1038/nrc2748)

Desiniotis A \& Kyprianou N 2011 Significance of talin in cancer progression and metastasis. International Review of Cell and Molecular Biology 289 117-147. (https://doi.org/10.1016/B978-0-12-3860392.00004-3)

Dralle H \& Machens A 2008 Surgical approaches in thyroid cancer and lymph-node metastases. Best Practice and Research Clinical Endocrinology and Metabolism 22 971-987. (https://doi.org/10.1016/j. beem.2008.09.018)
2018 Society for Endocrinology Published by Bioscientifica Ltd. Printed in Great Britain 
Fabien N, Fusco A, Santoro M, Barbier Y, Dubois PM \& Paulin C 1994 Description of a human papillary thyroid carcinoma cell line. Morphologic study and expression of tumoral markers. Cancer $\mathbf{7 3}$ 2206-2212. (https://doi.org/10.1002/10970142(19940415)73:8<2206::AID-CNCR2820730828>3.0.CO;2-M)

Gao D, Vahdat LT, Wong S, Chang JC \& Mittal V 2012 Microenvironmental regulation of epithelial-mesenchymal transitions in cancer. Cancer Research 72 4883-4889. (https://doi. org/10.1158/0008-5472.CAN-12-1223)

Graauw MD \& Thompson CB 2010 Annexin A1 regulates TGF-beta signaling and promotes metastasis formation of basal-like breast cancer cells. PNAS 107 6340. (https://doi.org/10.1073/ pnas.0913360107)

Guo C, Liu S, Wang J, Sun MZ \& Greenaway FT 2013 ACTB in cancer. Clinica Chimica Acta 417 39-44. (https://doi.org/10.1016/j. cca.2012.12.012)

Henderson YC, Toro-Serra R, Chen Y, Ryu J, Frederick MJ, Zhou G, Gallick GE, Lai SY \& Clayman GL 2014 Src inhibitors in suppression of papillary thyroid carcinoma growth. Head and Neck 36 375-384. (https://doi.org/10.1002/hed.23316)

Hood JL, San RS \& Wickline SA 2011 Exosomes released by melanoma cells prepare sentinel lymph nodes for tumor metastasis. Cancer Research $\mathbf{7 1}$ 3792-3801. (https://doi.org/10.1158/0008-5472.CAN-10-4455)

Hoshino A, Costasilva B, Shen TL, Rodrigues G, Hashimoto A, Mark MT, Molina H, Kohsaka S, Giannatale AD \& Ceder S 2015 Tumour exosome integrins determine organotropic metastasis. Nature $\mathbf{5 2 7}$ 329-335. (https://doi.org/10.1038/nature15756)

Howlader N, Noone AM, Krapcho M, Neyman N, Aminou R, Altekruse SF, Kosary CL, Ruhl J, Tatalovich Z \& Cho H 2012 SEER Cancer Statistics Review, 1975-2009 (Vintage 2009. Populations). Bethesda, MD, USA: National Cancer Institute.

Ji S, Zhang B, Liu J, Qin Y, Liang C, Shi S, Jin K, Liang D, Xu W \& Xu H 2016 ALDOA functions as an oncogene in the highly metastatic pancreatic cancer. Cancer Letters 374 127-135. (https://doi. org/10.1016/j.canlet.2016.01.054)

Jin JK, Tien PC, Cheng CJ, Song JH, Huang C, Lin SH \& Gallick GE 2015 Talin1 phosphorylation activates $\beta 1$ integrins: a novel mechanism to promote prostate cancer bone metastasis. Oncogene 34 1811-1821. (https://doi.org/10.1038/onc.2014.116)

Kaigorodova EV, Zavyalova MV, Bogatyuk MV, Tarabanovskaya NA, Slonimskaya EM \& Perelmuter VM 2015 Relationship between the expression of phosphorylated heat shock protein beta-1 with lymph node metastases of breast cancer. Cancer Biomarkers 15 143-150. (https://doi.org/10.3233/CBM-140446)

Kitahara CM \& Sosa JA 2016 The changing incidence of thyroid cancer. Nature Reviews Endocrinology 12 1137-1142. (https://doi.org/10.1038/ nrendo.2016.110)

Lim LHK \& Pervaiz S 2007 Annexin 1: the new face of an old molecule. FASEB Journal 21 968. (https://doi.org/10.1096/fj.06-7464rev)

Lin JD, Fu SS, Chen JY, Lee CH, Chau WK, Cheng CW, Wang YH, Lin YF, Fang WF \& Tang KT 2016 Clinical manifestations and gene expression in patients with conventional papillary thyroid carcinoma carrying the BRAFV600E mutation and BRAF pseudogene. Thyroid 26 691-704. (https://doi.org/10.1089/ thy.2015.0044)

Liu Y, Gu Y \& Cao X 2015 The exosomes in tumor immunity. Oncoimmunology 4 e1027472. (https://doi.org/10.1080/21624 02X.2015.1027472)

Mutalib NSA, Othman SN, Yusof AM, Suhaimi SNA, Muhammad R \& Jamal R 2016 Integrated microRNA, gene expression and transcription factors signature in papillary thyroid cancer with lymph node metastasis. PeerJ 4 e2119. (https://doi.org/10.7717/peerj.2119)

Pályi I, Péter I, Daubner D, Vincze B\& Lõrincz I 1993 Establishment, characterization and drug sensitivity of a new anaplastic thyroid carcinoma cell line (BHT-101). Virchows Archiv B 63 263-269.

Pagni F, L'Imperio V, Bono F, Garancini M, Roversi G, De Sio G, Galli M, Smith AJ, Chinello C \& Magni F 2015 Proteome analysis in thyroid pathology. Expert Review of Proteomics 12 375-390. (https://doi.org/10 $.1586 / 14789450.2015 .1062369)$

Park SI, Zhang J, Phillips KA, Araujo JC, Najjar AM, Volgin AY, Gelovani JG, Kim SJ, Wang Z \& Gallick GE 2008 Targeting SRC family kinases inhibits growth and lymph node metastases of prostate cancer in an orthotopic nude mouse model. Cancer Research 68 3323-3333. (https://doi.org/10.1158/0008-5472.CAN-07-2997)

Peng Y, Li X, Wu M, Yang J, Liu M, Zhang W, Xiang B, Wang X, Li X, Li G, et al. 2012 New prognosis biomarkers identified by dynamic proteomic analysis of colorectal cancer. Molecular BioSystems $\mathbf{8}$ 3077-3088. (https://doi.org/10.1039/c2mb25286d)

Polyak K \& Weinberg RA 2009 Transitions between epithelial and mesenchymal states: acquisition of malignant and stem cell traits. Nature Reviews Cancer 9 265-273. (https://doi.org/10.1038/nrc2620)

Rocchi P, Beraldi E, Ettinger S, Fazli L, Vessella RL, Nelson C \& Gleave M 2005 Increased Hsp27 after androgen ablation facilitates androgenindependent progression in prostate cancer via signal transducers and activators of transcription 3-mediated suppression of apoptosis. Cancer Research 65 11083-11093. (https://doi.org/10.1158/00085472.CAN-05-1840)

Rusinek D, Swierniak M, Chmielik E, Kowal M, Kowalska M, Cyplinska R, Czarniecka A, Piglowski W, Korfanty J \& Chekan M 2015 BRAFV600E-associated gene expression profile: early changes in the transcriptome, based on a transgenic mouse model of papillary thyroid carcinoma. PLOS ONE 10 e0143688. (https://doi.org/10.1371/ journal.pone.0143688)

Schneider DF \& Chen H 2013 New developments in the diagnosis and treatment of thyroid cancer. CA: A Cancer Journal for Clinicians 63 374-394. (https://doi.org/10.3322/caac.21195)

Schneider DF, Mazeh H, Chen H \& Sippel RS 2013 Lymph node ratio predicts recurrence in papillary thyroid cancer. Oncologist 18 157-162. (https://doi.org/10.1634/theoncologist.2012-0240)

Summy JM \& Gallick GE 2003 Src family kinases in tumor progression and metastasis. Cancer and Metastasis Reviews 22 337. (https://doi. org/10.1023/A:1023772912750)

von Thun A, Birtwistle M, Kalna G, Grindlay J, Strachan D, Kolch W, von Kriegsheim A \& Norman JC 2012 ERK2 drives tumour cell migration in three-dimensional microenvironments by suppressing expression of Rab17 and liprin-beta2. Journal of Cell Science $\mathbf{1 2 5}$ 1465-1477. (https://doi.org/10.1242/jcs.092916)

Wang C, Yan G, Zhang Y, Jia X \& Bu P 2015 Long non-coding RNA MEG3 suppresses migration and invasion of thyroid carcinoma by targeting of Rac1. Neoplasma 62 541-549. (https://doi.org/10.4149/ neo_2015_065)

Yang X, Sun J, Xia D, Can X, Liu L, Zhang J, Xu H, Du N, Liu W, Shen F, et al. 2017 Capn4 enhances osteopontin expression through activation of the Wnt/beta-catenin pathway to promote epithelial ovarian carcinoma metastasis. Cellular Physiology and Biochemistry 42 185-197. (https://doi.org/10.1159/000477310)

Zhen C, Chen L, Zhao Q, Liang B, Gu YX, Bai ZF, Wang K, Xu X, Han QY, Fang DF, et al. 2013 Gankyrin promotes breast cancer cell metastasis by regulating Rac1 activity. Oncogene 32 3452-3460. (https://doi.org/10.1038/onc.2012.356)

Received in final form 6 June 2018

Accepted 11 June 2018

Accepted Preprint published online 12 June 2018 (c) 2018 Society for Endocrinology Published by Bioscientifica Ltd. Printed in Great Britain 